\title{
Symptoms, Illness Perceptions, Self-Efficacy and Health-Related Quality of Life Following Colorectal Cancer Treatment
}

\author{
Ann-Caroline Johansson ${ }^{*}$, Malin Axelsson ${ }^{2}$, Gunne Grankvist ${ }^{3}$, Ina Berndtsson ${ }^{1}$, Eva Brink ${ }^{1}$ \\ ${ }^{1}$ Department of Health Sciences, University West, Trollhättan, Sweden \\ ${ }^{2}$ Department of Care Science, Faculty of Health and Society, Malmö University, Malmö, Sweden \\ ${ }^{3}$ Department of Social and Behavioural Studies, University West, Trollhättan, Sweden \\ Email: *ann-caroline.johansson@hv.se
}

How to cite this paper: Johansson, A.-C., Axelsson, M., Grankvist, G., Berndtsson, I. and Brink, E. (2018) Symptoms, Illness Perceptions, Self-Efficacy and Health-Related Quality of Life Following Colorectal Cancer Treatment. Open Journal of Nursing, $\mathbf{8}$, 591-604.

https://doi.org/10.4236/ojn.2018.89044

Received: August 9, 2018

Accepted: September 8, 2018

Published: September 11, 2018

Copyright (ㅇ 2018 by authors and Scientific Research Publishing Inc. This work is licensed under the Creative Commons Attribution International License (CC BY 4.0).

http://creativecommons.org/licenses/by/4.0/

\begin{abstract}
Introduction: Lower health-related quality of life (HRQoL) is associated with fatigue, poor mental and poor gastrointestinal health during the first three months after colorectal cancer (CRC) treatment. Research indicates that maintaining usual activities has a positive impact on HRQoL after treatment for CRC. Illness perceptions have been associated with HRQoL in other cancer diseases, and self-efficacy has been associated with HRQoL in gastrointestinal cancer survivors. Our knowledge about illness perceptions and selfefficacy in relation to maintaining everyday activities and HRQoL following CRC treatment is incomplete. Aim: To explore associations between HRQoL, fatigue, mental health, gastrointestinal health, illness perceptions and selfefficacy in relation to maintaining everyday activities, three months after surgical CRC treatment. A further aim was to test the Maintain Function Scale in a CRC population. Method: The study was cross-sectional. Forty-six persons participated. Data were collected using questionnaires. Descriptive and analytical statistics were used. Results: Persons who were more fatigued, depressed, worried, and had more diarrhea were more likely to report lower HRQoL. Increased fatigue and diarrhea were associated with decreased HRQoL. Concerning illness perceptions, persons who reported negative emotions and negative consequences of CRC were more likely to report lower HRQoL. Persons scoring higher on self-efficacy were more likely to report higher HRQoL. Increased self-efficacy was associated with increased HRQoL. The Maintain Function Scale was suitable for assessing self-efficacy in relation to maintaining everyday activities. Conclusions: Nursing support to improve self-efficacy and illness perceptions and to minimize symptoms during recovery should have a favorable impact on HRQoL.
\end{abstract}




\section{Keywords}

Colorectal Cancer, Health-Related Quality of Life, Illness Perceptions, Recovery, Self-Efficacy

\section{Introduction}

Health-related quality of life (HRQoL) is a patient-reported outcome measure commonly used after cancer therapy. HRQoL concerns the effects of disease and treatment on physical, psychological, and social wellbeing, including measures of symptoms [1]. Early recovery after colorectal cancer (CRC) treatment can be challenging and demanding in many respects. A temporary or permanent colostomy may have been required, and radiation and chemotherapy may also have been administered as supplementary treatments [2]. In addition to treatment-related effects, a health threat posed by CRC may negatively affect mental health by creating anxiety and depression after treatment [3]. Research shows a decrease in HRQoL during the first three months of recovery. Fatigue, poor mental health, constipation and diarrhea are associated with this decrease [4] [5]. Conversely, some aspects, such as maintaining social and other activities after treatment, have been shown to protect against depression and reduce emotional distress [6] [7].

Persons suffering from symptoms and other illness experiences caused by severe illness, such as CRC, create an image of their illness, and these images are defined as illness perceptions [8]. Illness perceptions are personal, cognitive representations of a disease and a parallel emotional response, i.e., emotional representation [8]. Illness perceptions are organized in a pattern, consisting of disease dimensions such as the consequences of the disease for everyday life, the emotional influence the disease has on life, the nature of the disease, and the expected duration of the disease [9]. Research on illness perception in relation to HRQoL in recovery shows that illness perceptions contribute to HRQoL in survivors of breast, colorectal and prostate cancer [10]. In breast cancer, perceiving less severe consequences has been associated with better HRQoL [11], and in head and neck cancer, negative emotional representations (i.e., perceived negative emotions associated with an illness) have been associated with poorer HRQoL [12]. Additionally, research shows that cancer patients who experience their cancer as emotionally difficult and as having negative consequences also perceive their cancer as more chronic [13]. In sum, research indicates that illness perceptions - i.e., consequences and emotional representations - are dimensions of illness perceptions that could be important for HRQoL during early recovery following CRC treatment.

Apart from illness perceptions, beliefs about self-efficacy could be important for HRQoL in recovery. General self-efficacy concerns people's assessment of their capability, not their actual capability per se [14]. The importance of 
self-efficacy for symptom and illness management in patients with cardiac disease [15] is well known. Among cancer survivors in general, connections have been found between higher self-efficacy and higher physical and psychological wellbeing [16]. In gastrointestinal cancer survivors, high self-efficacy in relation to illness behavior has been associated with better HRQoL [17]. Among colorectal cancer survivors, self-efficacy in relation to handling symptoms contributed to wellbeing in recovery [18]. Although early recovery is a time when fatigue, mental, and gastrointestinal health problems are common [4] [5], maintaining everyday activities, such as social and other activities [6] [7], and maintaining sexual [19] and physical activity after CRC treatment [20] have been shown to have a positive influence on wellbeing. In sum, research indicates that self-efficacy in relation to maintaining usual activities may be important for HRQoL after CRC treatment. No questionnaires are available specifically designed to measure self-efficacy in relation to maintaining everyday activities in persons recovering from CRC. Thus, there is a need for a questionnaire that takes these aspects into consideration. The Maintain Function Scale [21] would seem to be suitable for measuring self-efficacy in relation to maintaining everyday activities in the present sample of persons treated for CRC. The Maintain Function Scale consists of five items intended to assess a person's confidence in maintaining social and physical activities after experiencing a life-threatening disease.

The study aim was to explore associations between HRQoL, fatigue, mental health, gastrointestinal health, illness perceptions, i.e. consequences and emotional representations, and self-efficacy in relation to maintaining everyday activities three months after surgical CRC treatment. A further aim was to test the Maintain Function Scale in a CRC population.

\section{Materials and Methods}

\subsection{Sample and Setting}

Participants were selected using a consecutive sampling procedure [22]; over a 15-month period between March 2011 and June 2012, all patients surgically treated for CRC at a county hospital in western Sweden were invited to participate. A total of 81 patients were invited and 46 participated, resulting in a response rate of $57 \%$. The sampling procedure is shown in Figure 1. Participants were informed about the study verbally and in writing at the admission visit at the hospital ward. Study participants gave their written informed consent to participate, and with their consent, their medical records were accessed. All participants were guaranteed that their participation could be discontinued at any time without influencing their care. The study was approved by the Regional Ethical Review Board of Gothenburg (Reg. no. 753-10) before the data were collected.

\subsection{Data Collection}

The present study had a cross-sectional design. The data were gathered using 


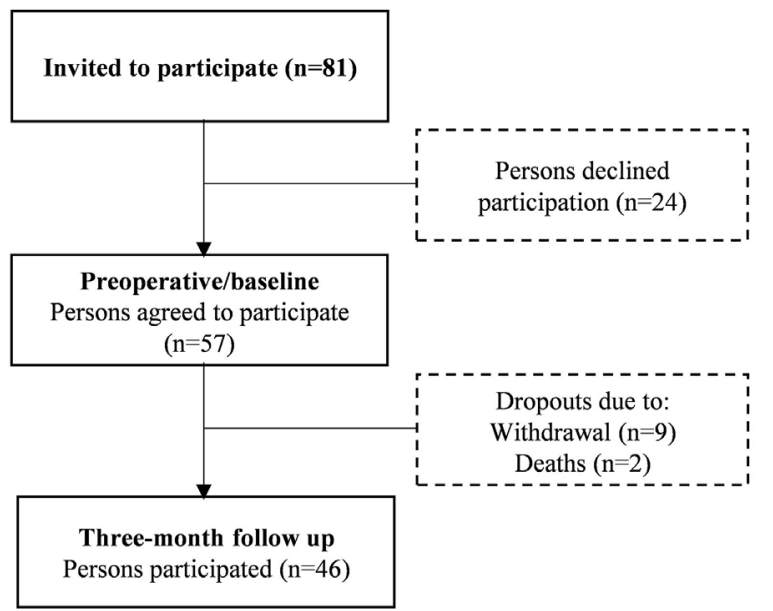

Figure 1. Flowchart of the sampling procedure $(n=46)$.

postal questionnaires, mailed on one occasion: three months post-surgical treatment. A package containing information, questionnaires and a prepaid envelope was sent to the participants' home address. Two reminder notifications were sent two weeks apart, after which time non-response was considered a withdrawal from the study. Data on medical and treatment-related information, such as the diagnoses cancer coli and cancer recti, stoma, additional treatment and complications, were later gathered from the medical records.

\subsection{Instruments}

The European Organization for Research and Treatment of Cancer's (EORTC) questionnaire QLQ-C30 (version 3.0) is a 30-item core instrument measuring HRQoL in persons affected by cancer diseases. QIQ-C30 incorporates one scale concerning general health and overall quality of life called the global health status/quality of life scale (GHS/QoL; here referred to as HRQoL), five function scales, three symptom scales and six single-symptom items [1]. In accordance with the aim of the present study, the GHS/QoL subscale (number of items = 2), the fatigue subscale (number of items $=3$ ), and the subscales for constipation and diarrhea (single-item symptom scales) were selected and used. The GHS/QoL items were scored from $1=$ very poor to $7=$ excellent. The items in all symptom scales were scored from $1=$ not at all to $4=$ very much. Scores on each scale were transformed into scores ranging from 0 to 100 according to the scoring manual [23]. High functional scores represent better functioning/HRQoL, while high symptom scores are related to more severe symptoms. The Swedish version used in the present study has been found to be valid and reliable [1] [24] [25]. In the present study, Cronbach's $\alpha$ ranged from 0.87 to 0.97 for the scales used.

The Hospital Anxiety and Depression Scale (HADS) was used to assess mental health. The scale is composed of 14 items divided into the subscales anxiety and depression; each item is rated on a 4 -point scale from $0=$ not at all to $3=$ mostly. Scores were summarized and ranged from $0-21$ points [26]. The HADS has been 
used in numerous studies, both with general and specific populations, and has been found to be a valid and reliable instrument [27]. The Swedish version used here was validated in a general population in 1997 [28]. In the present study, Cronbach's $\alpha$ for the depression scale was 0.80 and for the anxiety scale 0.88 .

The Illness Perception Questionnaire-Revised (IPQ-R) is a generic questionnaire that was used to measure perceptions of CRC. In the present study, the subscales Consequences (i.e., perceived negative impact of CRC on one's personal life) and Emotional representations (i.e., perceived negative emotions associated with the illness) were selected and used. The responses were made on a 5 -point scale ranging from $0=$ strongly disagree to $5=$ strongly agree. Scores were summarized and ranged from 0 to 30 . Higher scores on the consequences scale represent a more negative impact of CRC on personal life; higher scores on the emotional representations scale represent more negative emotions associated with the CRC [29]. The factor structure of the IPQ- $R$ has been confirmed in previous research in a range of conditions, including cancer populations [30]. The Swedish version used in the present study has been validated in patients with myocardial infarction [31]. In the current study, Cronbach's $\alpha$ for the consequences scale was 0.59 and for the emotional representation scale 0.79 .

The Swedish version of the Maintain Function Scale [32], which is one dimension of the Cardiac Self-Efficacy Scale, originally developed by Sullivan and colleagues [21] to measure self-reported self-efficacy in people with coronary heart disease, was used to measure self-efficacy in relation to maintaining everyday activities. This scale consists of five items of a general nature (Item $9-13$ on the cardiac self-efficacy scale) that assess aspects of daily life. How confident are you that you can: "Maintain your usual social activities," "maintain your usual activities at home," "maintain your usual activities outside your home," "maintain your sexual activities" and "get regular aerobic exercise". The responses were made on a 5 -point scale from $0=$ not at all confident to $4=$ completely confident. Scores were summarized and ranged from 0 to 20. A higher score indicates greater self-efficacy in relation to maintaining everyday activities [21]. The Cronbach's $\alpha$ for the scale was 0.95 .

\subsection{Analysis}

Descriptive statistics including frequencies, percentages, mean scores and standard deviations (SD) were calculated. Pearson's correlations were used to identify relationships between the variables. In addition, a multiple regression model, as presented by Pallant [33], was performed to identify predictors of HRQoL. All independent variables associated with HRQoL $(p<0.01)$ were included in the multiple regression model. Data analyses were performed in SPSS version 21 for Windows.

An exploratory factor analysis, also referred to as principal components analysis, was performed [33]. If one underlying factor can be identified that explains the variation in the five items, at a level of at least $60 \%$, the scale as a whole can be considered to measure one factor [34]. In the present study, self-efficacy 
measured by Maintain Function Scale was evaluated to see whether it worked as a one-dimensional scale in a sample of persons treated for CRC. The strength of underlying factors is assessed based on their eigenvalue. The factor with the highest eigenvalue explains the largest proportion of the variance in the material. The factor analysis involved the following steps: 1) Assessment of the suitability of the data by considering the sample size and the relationship between the items. According to Tabachnick and Fidell [35], a sample size corresponding to 5 cases per item and showing inter-item correlation coefficients above 0.3 is adequate. 2) Factor extraction, which involves determining the smallest number of factors that best describe the relationship between the items, here using Kaiser's criterion or the rule of eigenvalue greater than one. Only factors with an eigenvalue of one or more are kept.

\section{Results}

\subsection{Background Characteristics}

The characteristics of the participants are shown in Table 1. The participants consisted of 20 women and 26 men, with a mean age of 72.6 years. Twenty-four persons were diagnosed with cancer coli and 22 persons with cancer recti. Eleven persons had complications such as bleeding, dehiscence, abscess, anastomotic leakage, ileus, bladder dysfunction and infections within 30 days after surgery. None of the participants were diagnosed with metastatic disease at the time of data collection. Mean scores and standard deviations (SD) for HRQoL, symptoms, i.e. fatigue, constipation and diarrhea; mental health, i.e. depression and anxiety; illness perceptions, i.e. consequences and emotional representations; and self-efficacy in relation to maintaining everyday activities are presented in Table 2.

\subsection{Associations between Investigated Variables}

Fatigue, depression, anxiety, diarrhea, and illness perceptions (emotional representations and consequences) showed negative correlations with HRQoL, meaning that persons who were more fatigued, depressed, worried, or had more diarrhea were more likely to report lower HRQoL. Concerning illness perceptions, the results showed that those who reported more negative emotions and negative consequences of CRC were more likely to report lower HRQoL. Self-efficacy in relation to maintaining everyday activities, as measured by the Maintain Function Scale, showed a positive correlation with HRQoL, meaning that those who scored higher on such self-efficacy were more likely to report higher HRQoL, as shown in Table 3.

\subsection{Predictors of HRQoL}

The multiple regression model presented in Table 4 explained $78.8 \%$ of the variance in HRQoL (Adjusted R2 0.788, $\mathrm{p}<0.005$ ). Both fatigue and diarrhea were identified as negative predictors, indicating that an increase in these variables 
decreased HRQoL. Self-efficacy in relation to maintaining everyday activities was identified as a positive predictor, indicating that an increase in this variable increased HRQoL. However, diarrhea had a lower beta value compared to the other predictors, which suggests that its unique contribution to predicting HRQoL was smaller. In the present regression model, depression, anxiety, consequences, and emotional representations were not identified as significant predictors of HRQoL.

\subsection{Testing the Maintain Function Scale}

Because inter-item correlations were above 0.3 for all five items, (items ranged between 0.6 and 0.8 ), the data were determined to be suitable for factor analysis. The result of the factor analysis was based on an eigenvalue $>1$, which confirmed a one-factor solution. The first two eigenvalues were 3.96 and 0.43 . In accordance with the rule of eigenvalue $>1$, only the factor with such an eigenvalue was kept, and this one factor explained $79.16 \%$ of the variance in the total sample. The factor loading of each item of the Maintain Function Scale in the total sample is presented in Table 5 .

Table 1. Background characteristics of the study sample $(n=46)$.

\begin{tabular}{lc}
\hline Age mean (SD) & $72.6(7.32)$ \\
\hline Sex & $\mathrm{n}(\%)$ \\
Male & $26(56.5)$ \\
Female & $20(43.5)$ \\
Marital Status & $12(26.1)$ \\
Single & $34(73.9)$ \\
Married & \\
Work Status & $33(71.7)$ \\
Retired & $13(28.3)$ \\
Employed & \\
Diagnosis & $24(52.2)$ \\
Cancer coli & $22(47.8)$ \\
Cancer recti & \\
Stoma & $18(39.1)$ \\
Yes & $28(60.9)$ \\
No & \\
Chemotherapy & $7(15.2)$ \\
Yes & $39(84.8)$ \\
No & \\
Radiotherapy & $11(23.9)$ \\
Yes & $35(76.1)$ \\
No & \\
Complications & \\
No & \\
\hline & \\
\hline
\end{tabular}


A.-C. Johansson et al.

Table 2. Mean scores and standard deviations (SD) of investigated variables.

\begin{tabular}{lc}
\hline Scales & Mean scores (SD) \\
\hline HRQoL & $69.81(22.58)$ \\
Fatigue & $31.15(24.52)$ \\
Depression & $4.13(3.30)$ \\
Anxiety & $4.35(3.89)$ \\
Constipation & $13.04(24.82)$ \\
Diarrhea & $19.56(26.82)$ \\
Consequences & $16.93(3.89)$ \\
Emotional representations & $16.42(4.25)$ \\
$*$ Self-efficacy & $10.35(5.87)$ \\
\hline
\end{tabular}

*Self-efficacy in relation to maintaining everyday activities.

Table 3. Pearson's correlations between investigated variables.

\begin{tabular}{|c|c|c|c|c|c|c|c|c|}
\hline Variables & HRQoL & Fatigue & Depression & Anxiety & Constipation & Diarrhea & Consequences & $\begin{array}{c}\text { Emotional } \\
\text { representations }\end{array}$ \\
\hline Fatigue & $-0.803^{* *}$ & - & & & & & & \\
\hline Depression & $-0.707^{* *}$ & $0.719^{* *}$ & - & & & & & \\
\hline Anxiety & $-0.678^{\star *}$ & -0.100 & $0.649^{* *}$ & - & & & & \\
\hline Constipation & -0.170 & 0.210 & 0.089 & -0.021 & - & & & \\
\hline Diarrhea & $-0.392^{* *}$ & 0.266 & 0.187 & 0.103 & 0.218 & - & & \\
\hline Consequences & $-0.432^{\star *}$ & $0.394^{* *}$ & 0.195 & $0.429^{* *}$ & 0.170 & 0.275 & - & \\
\hline $\begin{array}{l}\text { Emotional } \\
\text { representations }\end{array}$ & $-0.553^{* *}$ & $0.464^{* *}$ & $0.460^{* *}$ & $0.665^{\star *}$ & 0.128 & 0.152 & $0.562^{\star *}$ & - \\
\hline${ }_{* * * \text { Self-efficacy }}$ & $0.722^{* *}$ & $-0.644^{* *}$ & $-0.623^{* *}$ & $-0.620^{\star *}$ & -0.209 & -0.274 & $-0.338^{\star}$ & $-0.535^{\star *}$ \\
\hline
\end{tabular}

${ }^{\star} \mathrm{P}<0.05 .{ }^{*} \mathrm{P}<0.01$. (2-tailed). ${ }^{* *}$ Self-efficacy in relation to maintaining everyday activities.

Table 4. Multiple regression model showing predictors of HRQoL as dependent variable $(n=46)$.

\begin{tabular}{lcc}
\hline & \multicolumn{2}{c}{ HRQoL $\left(\mathrm{R}^{2}{ }_{\text {adj }}=0.788\right) \mathrm{P}<0.005$} \\
\hline Variables & $\boldsymbol{\beta}$ & \multicolumn{1}{c}{ p-values } \\
\hline Fatigue & -0.385 & 0.002 \\
Depression & -0.108 & 0.363 \\
Anxiety & -0.126 & 0.293 \\
Diarrhea & -0.160 & 0.044 \\
Consequences & -0.002 & 0.983 \\
Emotional representations & 0.093 & 0.392 \\
${ }^{*}$ Self-efficacy & 0.292 & 0.012 \\
Sex & 0.078 & 0.320 \\
Age & 0.237 & 0.003 \\
\hline
\end{tabular}

${ }^{*}$ Self-efficacy in relation to maintaining everyday activities. 
Table 5. Factor loading of each item of the Maintain Function Scale in the total sample.

\begin{tabular}{lc}
\hline Item & Factor 1 \\
\hline 11. Maintain your usual activities outside your home & 0.951 \\
9. Maintain your usual social activities & 0.889 \\
10. Maintain your usual activities at home & 0.879 \\
12. Maintain your sexual activities & 0.867 \\
13. Get regular aerobic exercise & 0.860 \\
\hline
\end{tabular}

\section{Discussion}

Concerning symptoms and mental health, the results showed that persons who were more fatigued, depressed, worried or had more diarrhea were more likely to report lower HRQoL. The multiple regression model showed that an increase in symptoms such as fatigue and diarrhea decreased HRQoL in the early recovery phase after surgical treatment of CRC. Concerning illness perceptions, the results showed that those who reported negative emotions and negative consequences of CRC were more likely to report lower HRQoL. Concerning self-efficacy, the results showed that those who scored higher on self-efficacy in relation to maintaining everyday activities were more likely to report higher HRQoL. The multiple regression model showed that an increase in self-efficacy in relation to maintaining everyday activities was related to increased HRQoL in the early recovery phase.

Mental health, i.e. depression and anxiety, was negatively associated with HRQoL, meaning that persons who were more depressed or worried were more likely to have lower HRQol. These associations between anxiety (essentially depression) and HRQoL are in accordance with earlier findings [36]. However, in the present results, depression and anxiety did not predict HRQoL, which is inconsistent with findings from previous research [37]. Although mental health did not predict HRQoL here, and as suggested by previous research [37], mental health is important in recovery after CRC, and nurses therefore need to be attentive to mental health following CRC treatment. Among the studied symptoms, fatigue made the strongest unique contribution to HRQoL in the present results, meaning that more severe fatigue predicts poorer HRQoL. This is consistent with research showing that fatigue negatively influences HRQoL in persons experiencing CRC [38]. Diarrhea was also negatively associated with HRQoL. The impact of diarrhea on HRQoL found in the present study was not entirely unexpected, as diarrhea is a common symptom during early recovery and is known to negatively affect HRQoL [5]. The present results suggest that both fatigue and diarrhea need to be identified and addressed as early as possible. The etiologies underlying fatigue after cancer treatment are complex, and a variety of causes may be present and need to be addressed. Koornstra and colleagues [39] suggested a care plan for facilitating fatigue management. Their care plan includes screening for fatigue-preferably at the admission visit, while undergoing treat- 
ment, and after treatment-assessing severity and evaluating causes of the fatigue and options for pharmacological and/or non-pharmacological interventions, as well as evaluating self-efficacy beliefs. Based on the present findings, it would seem to be important to manage diarrhea in a similar manner. In addition to pharmacological treatment, it is important to inform patients about diet management as well [40].

Concerning illness perceptions, the results showed that those who reported negative emotions and consequences of CRC were more likely to report lower HRQoL. The associations found in the present results are consistent with earlier findings, showing that perception of less severe consequences was associated with better HRQoL in persons with breast cancer [11], and that having negative emotional representations was associated with poorer HRQoL in persons with head and neck cancer [12]. However, in the present results, illness perceptions did not predict HRQoL, which is inconsistent with findings from previous research [10]. Although illness perceptions did not predict HRQoL here, as suggested by previous research [10], the present results did show that illness perceptions were important in recovery after CRC. The present results highlighted the importance of further exploring these variables to discover whether they change over time, as well as how they relate to HRQoL over time.

Self-efficacy in relation to maintaining everyday activities functioned as the variable with the second strongest unique contribution in predicting HRQoL after CRC treatment, meaning that an increase in such self-efficacy was associated with increased HRQoL during recovery. The results presented here are in line with HRQoL research findings among colorectal and gastrointestinal cancer survivors indicating that better self-efficacy promotes better wellbeing HRQoL [17] [18]. The importance of self-efficacy in relation to maintaining everyday activities for HRQoL adds new and valuable clinical knowledge. Given the results of a 6-month-long nurse-led intervention program influenced by Bandura's strategies for changing self-efficacy beliefs showing successful improvement of self-efficacy in relation to chemotherapy treatment in patients with CRC [41], it should be possible to improve self-efficacy in relation to maintaining everyday activities using a similar intervention. The present results showed that the Maintain Function Scale was suitable for assessing self-efficacy in relation to maintaining everyday activities in the present study. They also indicate that the Maintain Function Scale might be useful for estimating self-efficacy in relation to maintaining everyday activities among persons with CRC in clinical settings, because it is short and takes into account aspects found to be of importance to persons with CRC.

The strength of the present study is that it explores the suitability of the Maintain Function Scale in assessing self-efficacy in a new disease population and that it has clear clinical implications. First, nurses need to be attentive to mental and gastrointestinal health early in recovery following CRC treatment, using care plans to address symptoms such as fatigue and diarrhea as one way to 
promote better HRQoL. Second, persons with negative illness perceptions in relation to their emotions and perceived consequences need to be identified and supported by nurses early in the recovery phase. One way to identify these persons could be by asking questions about emotions and thoughts concerning their illness. Third, it is important to strengthen self-efficacy, because an increase in self-efficacy should increase HRQoL in during recovery. One way to strengthen self-efficacy could be through implementation of self-efficacy enhancing interventions [41]. In sum, the present results support the notion that persons in recovery following CRC treatment would benefit from nurse-led follow-up consultations focused on symptoms, emotions, and thoughts in relation to their illness and information on how to increase self-efficacy in relation to maintaining everyday activities.

The size of the study is small, which is a limitation. The recommendations for what is considered a minimum sample size in factor analysis vary [35] [42], and using the minimum sample size may not always be the most beneficial approach. In factor analysis, high values on communalities are of interest as well, because high values can outweigh a small sample size. If the communality value of each variable is above 0.60 (in the present study they ranged from 0.74 to 0.90 ), a small sample size does not have to be of concern [43]. Considering these aspects as well as results from previous research, we concluded that the associations examined here would be strong enough to be detected in a small sample. A sample calculation was not performed. Nevertheless, the small number of participants does limit the generalizability of the present results. It is therefore recommended that the Maintain Function Scale be tested in larger CRC groups and validated. Further research in this area should include larger sample sizes with young adults represented, as well as comparisons of prognosis and of persons with and without colostomy.

\section{Conclusion}

Nursing support intended to improve self-efficacy in relation to maintaining everyday activities and illness perceptions in persons treated for CRC as well as to help them minimize their symptoms (e.g., fatigue and diarrhea) would probably have a favorable impact on their HRQoL during the recovery phase. More research in this area is warranted.

\section{Conflicts of Interests}

None to declare.

\section{References}

[1] Aaronson, N.K., et al. (1993) The European Organization for Research and Treatment of Cancer QLQ-C30: A Quality-Of-Life Instrument for Use in International Clinic Trials in Oncology. Journal of the National Cancer Institute, 85, 365-376. https://doi.org/10.1093/jnci/85.5.365

[2] Turner, P.S., Burke, D. and Finan, P.J. (2013) Nonresectional Management of Colo- 
rectal Cancer: Multidisciplinary Factors that Influence Treatment Strategy. Colorectal Disease, 15, 569-575. https://doi.org/10.1111/codi.12314

[3] Medeiros, M., Oshima, C.T. and Forones, N.M. (2010) Depression and Anxiety in Colorectal Cancer Patients. Journal of Gastrointestinal Cancer, 41, 179-184. https://doi.org/10.1007/s12029-010-9132-5

[4] Tsunoda, A., Nakao, K., Hiratsuka, K., Tsunoda, Y. and Kusano, M. (2007) Prospective Analysis of Quality of Life in the First Year after Colorectal Cancer Surgery. Acta Oncologica, 46, 77-82. https://doi.org/10.1080/02841860600847053

[5] Theodoropoulos, G.E., Karantanos, T., Stamopoulos, P. and Zografos, G. (2013) Prospective Evaluation of Health-Related Quality of Life after Laparoscopic Colectomy for Cancer. Techniques in Coloproctology, 17, 27-38. https://doi.org/10.1007/s10151-012-0869-7

[6] Kurtz, M.E., Kurtz, J.C., Stommel, M., Given, C.W. and Given, B. (2002) Predictors of Depressive Symptomatology of Geriatric Patients with Colorectal Cancer: A Longitudinal View. Supportive Care in Cancer, 10, 494-501. https://doi.org/10.1007/s00520-001-0338-8

[7] Hodgkinson, K., Butow, P., Hobbs, K.M. and Wain, G. (2007) After Cancer: The Unmet Supportive Care Needs of Survivors and Their Partners. Journal of Psychosocial Oncology, 25, 89-104. https://doi.org/10.1300/J077v25n04_06

[8] Leventhal, H., et al. (1997) Illness Representations: Theoretical Foundations. In: Petrie, K.J. and Weinman, J., Eds., Perceptions of Health and Illness, Harwood Academic Publisher, London, 19-45.

[9] Petrie, K.J. and Weinman, J. (2006) Why Illness Perceptions Matter. Clinical Medicine, 6, 536-539. https://doi.org/10.7861/clinmedicine.6-6-536

[10] Ashley, L., Marti, J., Velikova, G. and Wright P. (2015) Illness Perceptions within 6 Months of Cancer Diagnosis Are an Independent Prospective Predictor of Health-Related Quality of Life 15 Months Post-Diagnosis. Psychooncology, 24, 1463-1470. https://doi.org/10.1002/pon.3812

[11] Jörgensen, I.L., Frederiksen, K., Boesen, E., Elsass, P. and Johansen, C. (2009) An Exploratory Study of Associations between Illness Perceptions and Adjustment and Changes after Psychosocial Rehabilitation in Survivors of Breast Cancer. Acta Oncologica, 48, 1119-1127. https://doi.org/10.3109/02841860903033922

[12] Scharloo, M., et al. (2005) Quality of Life and Illness Perceptions in Patients with Recently Diagnosed Head and Neck Cancer. Head \& Neck, 27, 857-863. https://doi.org/10.1002/hed.20251

[13] Hopman, P. and Rijken, M. (2015) Illness Perceptions of Cancer Patients: Relationships with Illness Characteristics and Coping. Psycho-Oncology, 24, 11-18. https://doi.org/10.1002/pon.3591

[14] Bandura, A. (1997) General Self-Efficacy: The Exercise of Control. WH Freeman, New York.

[15] Clark, N.M. and Dodge, J.A. (1999) Exploring Self-Efficacy as a Predictor of Disease Management. Health Education and Behavior, 6, 72-89. https://doi.org/10.1177/109019819902600107

[16] Nelson, C.Y., Qian, L. and Wenjuan, L. (2014) Specificity May Count: Not Every Aspect of Coping Self-Efficacy Is Beneficial to Quality of Life among Chinese Cancer Survivors in China. International Journal of Behavioral Medicine, 21, 629-637. https://doi.org/10.1007/s12529-014-9394-6

[17] Kohno, Y., et al. (2010) Relationship of Psychological Characteristics and Self-Efficacy 
in Gastrointestinal Cancer Survivors. Psycho-Oncology, 19, 71-76. https://doi.org/10.1002/pon.1531

[18] Foster, C., et al. (2016) Pre-Surgery Depression and Confidence to Manage Problems Predict Recovery Trajectories of Health and Wellbeing in the First Two Years Following Colorectal Cancer: Results from the CREW Cohort Study. PLoS ONE, 1, e0155434. https://doi.org/10.1371/journal.pone.0155434

[19] Breukink, S.O. and Donovan, K.A. (2013) Physical and Psychological Effects of Treatment on Sexual Functioning in Colorectal Cancer Survivors. Journal of Sexual Medicine, 10, 74-83. https://doi.org/10.1111/jsm.12037

[20] Lin, K.Y., et al. (2014) Comparison of the Effects of a Supervised Exercise Program and Usual Care in Patients with Colorectal Cancer Undergoing Chemotherapy. Cancer Nursing, 37, 21-29. https://doi.org/10.1097/NCC.0b013e3182791097

[21] Sullivan, M.D., LaCroix, A.Z., Russo, J. and Katon, W.J. (1998) Self-Efficacy and Self-Reported Functional Status in Coronary Heart Disease: A Six-Month Prospective Study. Psychosomatic Medicine, 60, 473-478. https://doi.org/10.1097/00006842-199807000-00014

[22] Polit, D. and Beck, C. (2010) Essentials of Nursing Research: Appraising Evidence for Nursing Practice. 7th Edition, Lippincott Williams \& Wilkins, Philadelphia.

[23] Fayers, P.M., et al. (2001) The EORTC QLQ-C30 Scoring Manual. 3rd Edition, European Organisation for Research and Treatment of Cancer, Brussels.

[24] Bjordal, K., et al. (2000) A 12 Country Field Study of the EORTC QLQ-C30 (Version 3.0) and the Head and Neck Cancer Specific Module (EORTC QLQ-H\&N35) in Head and Neck Patients. EORTC Quality of Life Group. European Journal of Cancer, 36, 1796-1807. https://doi.org/10.1016/S0959-8049(00)00186-6

[25] Velikova, G., et al. (2012) Health-Related Quality of Life in EORTC Clinical Trials-30 Years Progress from Methodological Developments to Making a Real Impact on Oncology Practice. European Organisation for Research and Treatment of Cancer, 10, 141-149.

[26] Zigmond, A.S. and Snaith, R.P. (1983) The Hospital Anxiety and Depression Scale. Acta Psychiatrica Scandinavica, 67, 361-370. https://doi.org/10.1111/j.1600-0447.1983.tb09716.x

[27] Bjelland, I., Dahl, A.A., Haug, T.T. and Neckelmann, D. (2002) The Validity of the Hospital Anxiety and Depression Scale. An Updated Literature Review. Journal of Psychosom Research, 52, 69-77. https://doi.org/10.1016/S0022-3999(01)00296-3

[28] Lisspers, J., Nygren, A. and Soderman, E. (1997) Hospital Anxiety and Depression Scale (HAD): Some Psychometric Data for a Swedish Sample. Acta Psychiatrica Scandinavica, 96, 281-286. https://doi.org/10.1111/j.1600-0447.1997.tb10164.x

[29] Moss-Morris, R., Weinman, J., Petrie, K., Cameron, L. and Buick, D. (2002) The Revised Illness Perception Questionnaire. Psychology \& Health, 17, 1-16. https://doi.org/10.1080/08870440290001494

[30] Dempster, M. and McCorry, N.K. (2012) The Factor Structure of the Revised Illness Perception Questionnaire in a Population of Oesophageal Cancer Survivors. Psycho-Oncology, 21, 524-530. https://doi.org/10.1002/pon.1927

[31] Brink, E., Alsen, P. and Cliffordson, C. (2011) Validation of the Revised Illness Perception Questionnaire (IPQ-R) in a Sample of Persons Recovering from Myocardial Infarction-The Swedish Version. Scandinavian Journal of Psychology, 52, 573-579. https://doi.org/10.1111/j.1467-9450.2011.00901.x

[32] Fors, A., Ulin, K., Cliffordson, C., Ekman, I. and Brink, E. (2015) The Cardiac 
Self-Efficacy Scale, a Useful Tool with Potential to Evaluate Person-Centred Care. European Journal of Cardiovascular Nursing, 14, 536-543. https://doi.org/10.1177/1474515114548622

[33] Pallant, J. (2013) SPSS Survival Manual. A Step by Step Guide to Data Analysis Using IBM SPSS. Open University Press, Berkshire.

[34] Hair, J.F.J., Black, W.C., Babin, B.J., Anderson, R.E. and Tatham, K.L. (2006) Multivariate Data Analysis.

[35] Tabachnick, B.G. and Fidell, L.S. (2013) Using Multivariate Statistics. 6th Edition, Pearson Education, Boston.

[36] Tsunoda, A., et al. (2005) Anxiety, Depression and Quality of Life in Colorectal Cancer Patients. International Journal of Clinical Oncology, 10, 411-417. https://doi.org/10.1007/s10147-005-0524-7

[37] Pereira, M.G., Figueiredo, A.P. and Fincham, F.D. (2011) Anxiety, Depression, Traumatic Stress and Quality of Life in Colorectal Cancer after Different Treatments: A Study with Portuguese Patients and Their Partners. European Journal of Oncology Nursing, 16, 227-232. https://doi.org/10.1016/j.ejon.2011.06.006

[38] Marventano, S., et al. (2013) Health Related Quality of Life in Colorectal Cancer Patients: State of the Art. BMC Surgery, 13, S15. https://doi.org/10.1186/1471-2482-13-S2-S15

[39] Koornstra, R.H., Peters, M., Donofrio, S., van den Borne, B. and de Jong, F.A. (2014) Management of Fatigue in Patients with Cancer-A Practical Overview. Cancer Treatment Reviews, 40, 791-799. https://doi.org/10.1016/j.ctrv.2014.01.004

[40] Brant, J. and Walton, A. (2010) Chronic Diarrhea in Post-Treatment Colorectal Cancer Survivors. http://www.cancernetwork.com

[41] Zhang, M., et al. (2015) The Effectiveness of a Self-Efficacy-Enhancing Intervention for Chinese Patients with Colorectal Cancer: A Randomized Controlled Trial with 6-Month Follow up. International Journal of Nursing Studies, 51, 1083-1092. https://doi.org/10.1016/j.ijnurstu.2013.12.005

[42] Gorsuch, R.L. (1983) Factor Analysis. 2nd Edition, NJ Erlbaum, Hillsdale.

[43] Preacher, K.J. and MacCallum, R.C. (2002) Exploratory Factor Analysis in Behavior Genetics Research: Factor Recovery with Small Sample Sizes. Behavior Genetics, 32, 153-161. https://doi.org/10.1023/A:1015210025234 